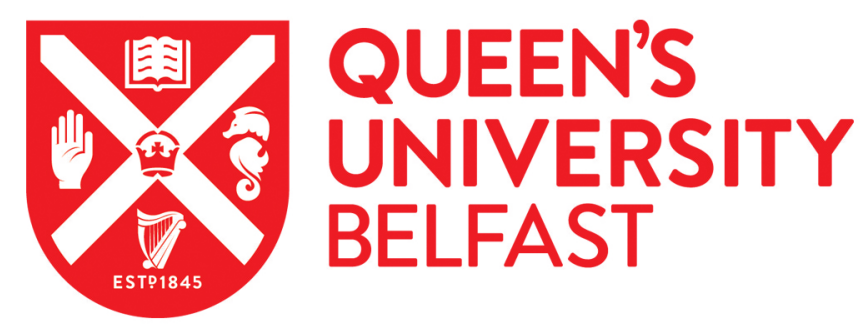

\title{
A Closed-Form Symbol Error Probability for MCIK-OFDM with Frequency Diversity
}

Luong, T. V., \& Ko, Y. (2017). A Closed-Form Symbol Error Probability for MCIK-OFDM with Frequency Diversity. In IEEE 18th International Worksop on Signal Processing Advances in Wireless Communications, SPAWC 2017 [17451509] Institute of Electrical and Electronics Engineers Inc.. https://doi.org/10.1109/SPAWC.2017.8227776

Published in:

IEEE 18th International Worksop on Signal Processing Advances in Wireless Communications, SPAWC 2017

Document Version:

Peer reviewed version

Queen's University Belfast - Research Portal:

Link to publication record in Queen's University Belfast Research Portal

Publisher rights

@ 2017 IEEE. This work is made available online in accordance with the publisher's policies. Please refer to any applicable terms of use of the publisher.

\section{General rights}

Copyright for the publications made accessible via the Queen's University Belfast Research Portal is retained by the author(s) and / or other copyright owners and it is a condition of accessing these publications that users recognise and abide by the legal requirements associated with these rights.

Take down policy

The Research Portal is Queen's institutional repository that provides access to Queen's research output. Every effort has been made to ensure that content in the Research Portal does not infringe any person's rights, or applicable UK laws. If you discover content in the Research Portal that you believe breaches copyright or violates any law, please contact openaccess@qub.ac.uk. 


\title{
A Closed-Form Symbol Error Probability for MCIK-OFDM with Frequency Diversity
}

\author{
Thien Van Luong and Youngwook Ko \\ Institute of Electronics, Communications and Information Technology \\ Queens University of Belfast \\ Belfast, NI, BT3 9DT, United Kingdom \\ Email: \{tluong01,y.ko\}@qub.ac.uk
}

\begin{abstract}
This paper proposes a novel scheme of Multicarrier Index Keying - Orthogonal Frequency Division Multiplexing (MCIK-OFDM), called as repeated MCIK-OFDM (ReMO), which is capable of improving the error performance compared to classical MCIK-OFDM for a given spectral efficiency. Similar to the classical MCIK-OFDM, ReMO activates only a subset of sub-carriers to carry information bits by the use of both the active sub-carriers and their indices. However, unlike the classical scheme, ReMO exploits the frequency diversity such that the same $M$-ary data symbol is repeatedly used to modulate all the active sub-carriers. To gain an insight into the ReMO performance, a closed-form expression for the symbol error probability (SEP) is derived. This expression can help us analyze the impact of the ReMO parameters on the symbol error performance and suggest the best ReMO setup to minimize the error probability. Through both theoretical and simulation results, ReMO is successfully proved to provide significantly better diversity order as well as SEP error performance over the conventional OFDM and MCIK-OFDM at the same spectral efficiency.
\end{abstract}

Index Terms-MICK-OFDM, index modulation (IM), symbol error probability (SEP), frequency diversity, maximum likelihood (ML) detection.

\section{INTRODUCTION}

Multi-carrier Index Keying - Orthogonal Frequency Division Multiplexing (MCIK-OFDM) or its so-called OFDM-IM (Index Modulation) in [1] has been recently proposed as a new multi-carrier technique that extends the conventional two dimensional $\mathrm{M}$-ary signal constellations to a third dimension, which is the sub-carrier index. In MCIK-OFDM transmission, only a subset of sub-carriers is activated, according to the incoming data, to convey constellation symbols. The use of activated sub-carrier indices as an additional degree of freedom enables the transmission of extra information bits without any additional bandwidth and power requirements. This MCIKOFDM principle aims to improve the reliability and the energy efficiency for wireless systems [2].

The MCIK-OFDM has attracted significant attention because it can provide a balanced trade-off between error probability and spectral efficiency. Various MCIK-OFDM structures have been proposed and discussed to improve the performance. In [2], the tight upper bound on the bit error rate for MCIKOFDM has been first derived in closed-form. In [3], OFDM with in-phase/quadrature index modulation (OFDM-I/Q-IM) has been proposed, which applies the IM to both the inphase and the quadrature components of signals. In [4], a combination of MCIK-OFDM and space time block codes was proposed to achieve an additional coding gain. Meanwhile, the space-frequency coded index modulation (SFC-IM) scheme has been proposed in [5], which can attain a diversity order of 2 with the deployment of multiple-input multiple-output (MIMO) structure. The concept of MIMO-OFDM-IM was investigated in [6]. To increase the diversity order at low complexity MCIK-OFDM, in [7] the authors have proposed an MCIK-OFDM scheme using the maximal ratio combining (MRC) with the design of a low complexity detector called greedy detection. To enhance the spectral efficiency, in [8], a generalised MCIK-OFDM which has a variable number of active sub-carriers has been proposed and in [9]-[10], the authors have introduced the dual-mode OFDM scheme and its corresponding generalised version in order to improve the attainable throughput over the classical MCIK-OFDM. Very recently, in [11], [12], the compressed sensing technique for MCIK-OFDM has been addressed to achieve higher spectral efficiency and energy efficiency. However, for high reliability, it is motivated to develop a simple transmit diversity structure for MCIK-OFDM that can enhance the error performance without degrading the data rate. To the best of our knowledge, a frequency diversity has been little explored, and its unique properties of attaining higher diversity gain have not been analyzed in the literature.

The MCIK-OFDM can be considered under the scenarios of ultra-reliable machine type communications (MTC) [13], which require a low throughput, but an extremely high reliability and an ultra-low latency. In fact, most of the aforementioned papers have focused on the improvement in the spectral efficiency such as [8], [9], [10], [11], which may not be suitable for MTC applications being sensitive more to a very low error rate than a high data rate. While some researchers use quite complicated designs of transceivers like [4], [3], some efforts have been made to use multiple antennas to achieve a spatial diversity gain in [5], [6], [7]. However, these may not meet the requirement of a simple and flexible operation of MTC devices that are low-cost and too small to equip more than one antenna.

In this paper, a repeated MCIK-OFDM termed as ReMO is proposed, taking into account hybrid frequency-and-index diversity. We combine MCIK with frequency diversity to modulate a subset of sub-carriers with the same $M$-ary data symbol. This approach can benefit from the advantages of 
both MCIK and frequency diversity transmission. Particularly, by modulating part of sub-carriers and the indices with the same $M$-ary data symbols, ReMO can be used to attain both frequency diversity and index diversity, leading to a significant transmit diversity. Such a highly reliable multi-carrier wireless transmission is timely for wireless MTC systems in an OFDM structure such as. e.g., 3GPP's narrow bandwidth MTC (NBMTC) [14]. To this end, novel closed-form expressions for the instantaneous and average symbol error probability (SEP) are derived, which can help us gain an insight into the performance of the ReMO scheme, for various cases. The derived SEP expressions are generalized to account for any number of active sub-carriers and repeated patterns. This enables the SEP performance analysis of different ReMO concepts by investigating the effects of higher modulation constellation and repeated ratio. The numerical results will clearly show that the ReMO outperforms the benchmarks, achieving a significantly high reliability, which is suitable to MTC applications.

The rest of this paper is organised as follows. Section II describes the system model, while section III proposes a theoretical bound on the SEP and the performance analysis. The numerical results and discussion are presented in section $\mathrm{VI}$ and section $\mathrm{V}$ concludes the paper.

Notation: $C(k, n)$ and $\lfloor x\rfloor$ denote the binomial coefficient and the largest integer less than or equal to $x$, respectively. Column vectors and matrices are presented by bold lowercase and capital letters, respectively. $\mathbb{E}\{x\}$ and $\mathcal{M}_{x}($.$) denote$ the expectation and the moment generating function of the random variable $x$, respectively. $(.)^{T}$ and $\mathcal{C} \mathcal{N}($.$) denote the$ complex conjugation and the circularly-symmetric complex normal distribution, respectively.

\section{SySTEM MODEL}

Consider a multi-carrier wireless system with $N_{c}=G N$ sub-carriers, including $G$ blocks of $N$-sub-carriers. Like the classical MCIK-OFDM, we activate only $K$ out of the total $N$ sub-carriers in each block to carry data bits and the remaining inactive sub-carriers are zero padded. Denote by $s$ an $M$-ary constellation symbol. Different from the classical scheme, which sends different $M$-ary constellation symbols over $K$ active sub-carriers, we propose to repeatedly use the same $M$-ary constellation symbol $s$ in modulating $K \geq 2$ active sub-carriers for each MCIK-OFDM transmission. For convenience, this scheme is referred to as repeated MCIKOFDM (ReMO) throughout the paper. Such repeated mapping of a single $s$ to $K$ active sub-carriers in ReMO is expected to provide a diversity effect in both the frequency and index domains. Notice that such a mapping is independently applied to every block. For convenience and without loss of generality, hereinafter, we address only one block instead of the whole $G$ blocks.

For a ReMO block, in every symbol period, incoming $p_{1}+p_{2}$ bits are partitioned into 2 groups at the transmitter. The first $p_{1}$ bits are mapped to an index symbol, which is corresponding to a combination of $K$ active sub-carriers whose indices are in a set of $\theta$, i.e., $\theta=\left\{\alpha_{1}, . ., \alpha_{K}\right\}$, where $\alpha_{k} \in\{1, \ldots, N\}$. Here, the index symbol can be defined by a vector as $\boldsymbol{\lambda}=\left[\beta_{1}, \ldots, \beta_{N}\right]^{T}$, where $\beta_{i}=1$ if $i \in \theta$ and $\beta_{i}=0$ if $i \notin \theta$. For given $N$ and $K$, let $\Phi$ be the set of index symbols in total and its cardinality is given by $m_{1}=|\Phi|=2^{\left\lfloor\log _{2} C(K, N)\right\rfloor}$, leading to $p_{1}=\left\lfloor\log _{2} C(K, N)\right\rfloor$. In addition, the combinatorical method [1] is used to generate the $m_{1}$ index symbols for our scheme. The remaining $p_{2}$ bits $\left(p_{2}=\log _{2} M\right)$ are modulated by one $M$-ary symbol $s$. Accordingly, the ReMO codeword is defined as $\boldsymbol{x}$, which is given by $\boldsymbol{x}=\boldsymbol{\lambda} s$.

The received signal vector $\boldsymbol{y}$ can be, in the frequency domain, expressed as

$$
\begin{aligned}
\boldsymbol{y} & =\boldsymbol{H} \boldsymbol{x}+\boldsymbol{n} \\
& =\boldsymbol{H} \boldsymbol{\lambda} s+\boldsymbol{n},
\end{aligned}
$$

where $\boldsymbol{H}=\operatorname{diag}\{h(1), \ldots, h(N)\}$ denotes the channel matrix, where $h(\alpha)$ is a flat Rayleigh fading channel of subcarrier $\alpha$, being an independent, complex-valued Gaussian random variable with zero mean and unit variance, i.e., $h(\alpha) \sim \mathcal{C N}(0,1)$, and $\boldsymbol{n}=[n(1), . ., n(N)]^{T}$ is the additive Gaussian noise vector with its entries $n(\alpha) \sim \mathcal{C N}\left(0, N_{0}\right)$. For the power constraint, we assume that $\mathbb{E}\left\{\left|s^{2}\right|\right\}=\varphi E_{s}$, where $E_{s}$ and $\varphi=N / K$ are the transmit power per $M$-ary symbol $s$ and the power allocation coefficient, respectively. Hence, the average signal-to-noise ratio (SNR) per sub-carrier at the receiver is given by $\bar{\gamma}=\varphi E_{s} / N_{0}$.

Finally, to recover the transmitted signal, the maximum likelihood (ML) detector is employed to make a decision on all possible ReMO codewords consisting of both all possible index symbols $\boldsymbol{\lambda}$ and $M$-ary symbols $s$, and the estimate can be written as

$$
\hat{\boldsymbol{x}}=(\hat{\boldsymbol{\lambda}}, \hat{s})=\arg \min _{\boldsymbol{\lambda}, s}\|\boldsymbol{y}-\boldsymbol{H} \boldsymbol{\lambda} s\|^{2} .
$$

\section{ERror PERFormance ANALYSIS}

\section{A. Pairwise index error probability}

We first consider the index detection; the well-known conditional pairwise index-detection error probability (PEP) of ML can be used to represent the error event that the transmitted $i$-th index vector is incorrectly detected as $j$-th index vector. This can be given by

$$
P\left(\boldsymbol{\lambda}_{i} \rightarrow \boldsymbol{\lambda}_{j}\right)=Q\left(\sqrt{\frac{\varphi E_{s}\left\|\boldsymbol{H} \boldsymbol{\lambda}_{i}-\boldsymbol{H} \boldsymbol{\lambda}_{j}\right\|^{2}}{2 N_{0}}}\right),
$$

where $\boldsymbol{\lambda}_{i}$ and $\boldsymbol{\lambda}_{j}$ denote the transmitted index vector and the decoded index vector, respectively, and $Q(x)=$ $\frac{1}{2 \pi} \int_{x}^{\infty} \exp \left(-\frac{t^{2}}{2}\right) \mathrm{d} t$ is the Gaussian tail probability [15].

Let $\boldsymbol{\lambda}_{j}$ and $\boldsymbol{\lambda}_{i}$ have a Hamming distance of $2 D$. Then, PEP in (3) can be rewritten as

$$
P\left(\boldsymbol{\lambda}_{i} \rightarrow \boldsymbol{\lambda}_{j}\right)=Q\left(\sqrt{\frac{\sum_{d=1}^{D}\left(\gamma_{\alpha_{d}}+\gamma_{\tilde{\alpha}_{d}}\right)}{2}}\right)
$$

where $\alpha_{d} \in \theta_{i}$ and $\tilde{\alpha}_{d} \in \theta_{j}$ such that $\alpha_{d}, \tilde{\alpha}_{d} \notin \theta_{i} \cap \theta_{j}$, and $\gamma_{\alpha}=\bar{\gamma}|h(\alpha)|^{2}$ denotes the instantaneous SNR of sub-carrier $\alpha$. 
Using the union bound, the probability of the index misdetetion of $\boldsymbol{\lambda}_{i}$ can be obtained, for given $\boldsymbol{\lambda}_{i}$, as

$$
P E P_{i} \leq \sum_{j \neq i=1}^{m_{1}} P\left(\boldsymbol{\lambda}_{i} \rightarrow \boldsymbol{\lambda}_{j}\right)
$$

and, therefore, the instantaneous PEP of ReMO can be expressed as

$$
P E P=\frac{1}{m_{1}} \sum_{i=1}^{m_{1}} P E P_{i} \leq \frac{1}{m_{1}} \sum_{i=1}^{m_{1}} \sum_{j \neq i=1}^{m_{1}} P\left(\boldsymbol{\lambda}_{i} \rightarrow \boldsymbol{\lambda}_{j}\right) .
$$

Denote by $\Omega_{i}$ the set of indices $j(\neq i)$ such that $\boldsymbol{\lambda}_{j}$ is satisfying the Hamming distance of 2 with $\boldsymbol{\lambda}_{i}$ i.e., $D=1$ in (4). Denote by $\eta_{i}$ the cardinality of $\Omega_{i}$ i.e., $\eta_{i}=\left|\Omega_{i}\right|$. Based on (4) and (5), notice that $P E P_{i}$ is dominant with the following terms $P\left(\boldsymbol{\lambda}_{i} \rightarrow \boldsymbol{\lambda}_{j} \mid j \in \Omega_{i}\right)$, i.e., $\sum_{j \neq i=1}^{m_{1}} P\left(\boldsymbol{\lambda}_{i} \rightarrow \boldsymbol{\lambda}_{j}\right) \approx$ $\sum_{j \in \Omega_{i}} P\left(\boldsymbol{\lambda}_{i} \rightarrow \boldsymbol{\lambda}_{j}\right)$. Hence, (6) can be approximated by

$$
\begin{aligned}
P E P & \leq \frac{1}{m_{1}} \sum_{i=1}^{m_{1}} \sum_{j \in \Omega_{i}} P\left(\boldsymbol{\lambda}_{i} \rightarrow \boldsymbol{\lambda}_{j}\right) \\
& =\frac{1}{m_{1}} \sum_{i=1}^{m_{1}} \sum_{j \in \Omega_{i}} P(\alpha \rightarrow \tilde{\alpha}),
\end{aligned}
$$

where $P(\alpha \rightarrow \tilde{\alpha})=Q\left(\sqrt{\frac{\gamma_{\alpha}+\gamma_{\tilde{\alpha}}}{2}}\right)$ is the simplified pairwise probability of incorrectly detecting an active sub-carrier $\alpha \in$ $\theta_{i}$ into an inactive sub-carrier $\tilde{\alpha} \in \theta_{j}$, where $\theta_{i}, \theta_{j}$ are the corresponding index sets of $\boldsymbol{\lambda}_{i}, \boldsymbol{\lambda}_{j}$, respectively.

Then, the overall average PEP of ReMO can be given by

$$
\begin{aligned}
\overline{P E P} & \leq \frac{1}{m_{1}} \sum_{i=1}^{m_{1}} \sum_{j \in \Omega_{i}} \mathbb{E}_{\gamma_{\Sigma}}\left\{Q\left[\sqrt{\frac{\gamma_{\Sigma}}{2}}\right]\right\} \\
& \approx \mathbb{E}_{\gamma_{\Sigma}}\left\{\frac{\sum_{i=1}^{m_{1}} \eta_{i}}{m_{1}}\left(\frac{1}{12} e^{-\frac{\gamma_{\Sigma}}{4}}+\frac{1}{4} e^{-\frac{\gamma_{\Sigma}}{3}}\right)\right\},
\end{aligned}
$$

where $\gamma_{\Sigma}=\gamma_{\alpha}+\gamma_{\tilde{\alpha}}$ and we use the approximation of $Q(x) \approx$ $\frac{1}{12} e^{-\frac{x^{2}}{2}}+\frac{1}{4} e^{-\frac{2}{3} x^{2}}$.

Due to the definition of the moment generating function (MGF) [15], i.e., $\mathbb{E}_{\gamma}\left\{e^{-s \gamma}\right\}=\mathcal{M}_{\gamma}(s)$, the MGF of $\gamma_{\Sigma}$ can be calculated as $\mathcal{M}_{\gamma_{\Sigma}}(s)=\mathcal{M}_{\gamma}^{2}(s)=\frac{1}{(1-\bar{\gamma} s)^{2}}$. Finally, with the use of MGF approach, a closed-form expression for the approximate average PEP in (8) can be obtained as

$$
\begin{aligned}
\overline{P E P} & \leq \frac{\sum_{i=1}^{m_{1}} \eta_{i}}{12 m_{1}} \mathbb{E}_{\gamma_{\Sigma}}\left\{e^{-\frac{\gamma_{\Sigma}}{4}}+3 e^{-\frac{\gamma_{\Sigma}}{3}}\right\} \\
& =\frac{\sum_{i=1}^{m_{1}} \eta_{i}}{12 m_{1}}\left[\mathcal{M}_{\gamma_{\Sigma}}\left(-\frac{1}{4}\right)+3 \mathcal{M}_{\gamma_{\Sigma}}\left(-\frac{1}{3}\right)\right] \\
& =\frac{\sum_{i=1}^{m_{1}} \eta_{i}}{12 m_{1}}\left[\frac{16}{(4+\bar{\gamma})^{2}}+\frac{27}{(3+\bar{\gamma})^{2}}\right] .
\end{aligned}
$$

It is seen from (9) that the average PEP depends on $N, K$ through $\bar{\gamma}=N E_{s} / K N_{0}$ and $m_{1}=\lfloor C(K, N)\rfloor_{2^{p}}$. Moreover, for given $N$ and $K$, the PEP is also affected by the design of the index symbols $\lambda$ via the term of $\sum_{i=1}^{m_{1}} \eta_{i}$. Since ML detector exploits information from the indices of both active and inactive sub-carriers, it can be found from (9) that the achievable diversity order is 2, i.e., $-\lim _{\bar{\gamma} \rightarrow \infty}[\log (\overline{P E P}) / \log (\bar{\gamma})]=2$.

\section{B. Symbol error probability (SEP)}

A symbol error event occurs if either $\hat{\boldsymbol{\lambda}} \neq \boldsymbol{\lambda}$ or $\hat{s} \neq s$, thus, with each transmission of ReMO codeword consisting of two different symbols $(\boldsymbol{\lambda}$ and $s$ ), the number of potential symbol error events will be 0,1 or 2 . As a result, the instantaneous SEP and its average value can be respectively approximated by

$$
\begin{aligned}
S E P \leq \frac{1}{m_{1}} \sum_{i=1}^{m_{1}} \frac{1}{2}\left[P_{M}+\sum_{j \in \Omega_{i}} P(\alpha \rightarrow \tilde{\alpha})\right] \\
\overline{S E P} \leq \frac{\overline{P E P}+\bar{P}_{M}}{2},
\end{aligned}
$$

where $P_{M}$ is the instantaneous SEP of $M$-PSK constellations. The expression for $P_{M}$ is given by $P_{M} \approx$ $2 Q\left(\sqrt{2 \gamma_{\Sigma_{\alpha}}} \sin (\pi / M)\right)$ as in [16], where $\gamma_{\Sigma_{\alpha}}=\sum_{k=1}^{K} \gamma_{\alpha_{k}}$ and $\alpha_{k} \in \theta_{i}$. Next, using the approximation of $Q$-function as in (8), we can represent $P_{M}$ as follows

$$
P_{M} \approx \frac{1}{6}\left(e^{-\rho \gamma_{\Sigma_{\alpha}}}+3 e^{-\frac{4 \rho \gamma_{\Sigma_{\alpha}}}{3}}\right)
$$

where $\rho=\sin ^{2}(\pi / M)$. Similar to the previous subsection, utilizing the MGF method for the random variable $\gamma_{\Sigma_{\alpha}}$ that has the MGF of $\mathcal{M}_{\gamma_{\Sigma_{\alpha}}}(s)=\mathcal{M}_{\gamma}^{K}(s)=\frac{1}{(1-\bar{\gamma} s)^{K}}$, then, the average value of $P_{M}$ in (12) can be attained by

$$
\bar{P}_{M} \approx \frac{1}{6}\left[\frac{1}{(1+\rho \bar{\gamma})^{K}}+\frac{3}{\left(1+\frac{4 \rho \bar{\gamma}}{3}\right)^{K}}\right] .
$$

Finally, using (9), (11), and (13), the closed-form expression for the average SEP of ReMO is obtained as

$$
\begin{aligned}
\overline{S E P} & \leq \frac{\sum_{i=1}^{m_{1}} \eta_{i}}{24 m_{1}}\left[\frac{16}{(4+\bar{\gamma})^{2}}+\frac{27}{(3+\bar{\gamma})^{2}}\right] \\
& +\frac{1}{12}\left[\frac{1}{(1+\rho \bar{\gamma})^{K}}+\frac{3}{\left(1+\frac{4 \rho \bar{\gamma}}{3}\right)^{K}}\right] .
\end{aligned}
$$

It is seen from (14) that in the high SNR regions, the average SEP of ReMO mainly depends on the accuracy of the index detection as $K$ grows. This is because at very large $K$, the diversity order in (14) is always limited by 2 , that is exactly the diversity order attained in the index domain. By contrast, the diversity order of the classical MCIK-OFDM is limited to 1 since this scheme only uses the information of one sub-channel to detect the $M$-ary symbols. Thus, attaining diversity order twice higher than the classical scheme, ReMO is expected to enhance the average SEP performance.

In addition, for a given $N$, the ReMO system will provide the best SEP when $K$ is chosen not to be much greater than 2: $K=2,3$ can be the best choices to minimize the SEP. This will be verified by numerical results in the next section. 
It can also be found from (9) that $K$ should be chosen to be less than $N / 2$. For example, consider an ReMO scheme having $K>N / 2$ and another one with $K^{*}=N-K<N / 2$, it is obvious that these two values for $K$ provide the same spectral efficiency (due to $C(K, N)=C\left(K^{*}, N\right)$ ). However, the latter will result in a better error performance than the former because the latter has a much greater $\bar{\gamma}$ compared to the former, while the terms of $\sum_{i=1}^{m_{1}} \eta_{i}$ are nearly the same in both schemes.

\section{NUMERICAL RESULTS AND DISCUSSION}

In this section, we present the simulation and theoretical results for the average SEP of the proposed ReMO schemes with different parameters $K, N$ and $M$, over the flat Rayleigh fading condition. For comparison, we depict the results of the classical OFDM and MCIK-OFDM [1]. The SEP performance of these systems is evaluated by Monte Carlo simulations as a function of the average SNR per sub-carrier $E_{s} / N_{0}$.

\section{A. Comparison with classical OFDM and MCIK-OFDM}

We first compare the SEP performance curves of ReMO with classical OFDM and MCIK-OFDM at the same date rate of $1 \mathrm{bps} / \mathrm{Hz}$ (bits/s/Hz) as shown in Fig. 1. It is clearly observed from this figure that at a $S E P=10^{-4}$, ReMO provides considerable SNR gains of more than $14 \mathrm{~dB}$ and 9 $\mathrm{dB}$ over the classical OFDM and MCIK-OFDM, respectively. Even another ReMO scheme with a better date rate of 1.25 pbs/Hz in Fig. 1 also exhibits a much better SEP performance than its two classical counterparts with $12 \mathrm{~dB}$ and $7 \mathrm{~dB}$ SNR gains at $S E P=10^{-4}$. This impressive improvement is understandable because our novel scheme has the achievable diversity order twice that of classical schemes as presented in (14).

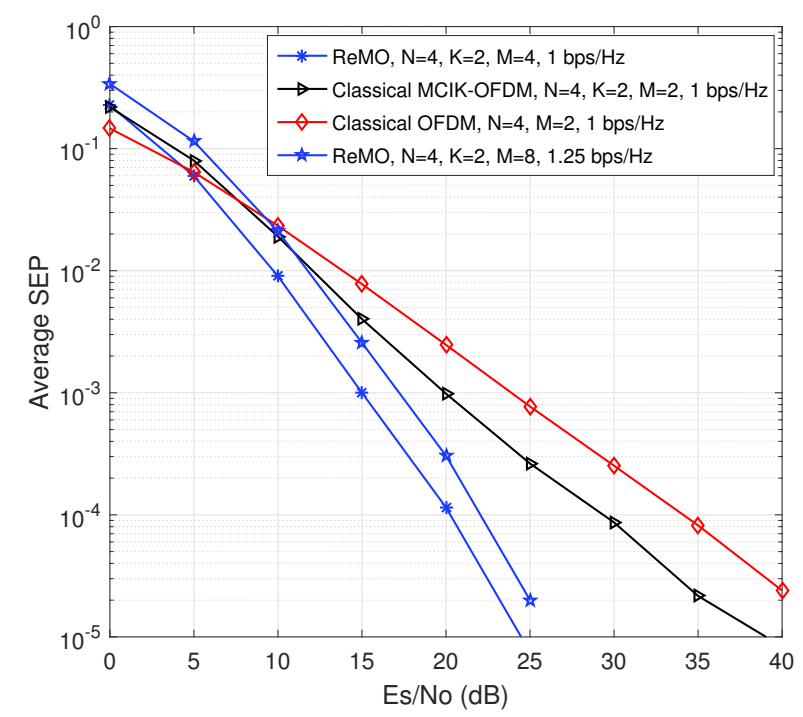

Fig. 1. Average SEP comparison of ReMO with both the conventional OFDM and MCIK-OFDM when $N=4, K=2, M \in\{2,4,8\}$.

\section{B. The tightness of the average SEP theoretical bound}

The tightness of the theoretical SEP expression derived in (14) is successfully verified in Fig. 2. As seen from this figure, the theoretical bounds for the SEP become very tight with increasing SNR values for all cases and the difference between bounds and simulations is negligible if the SEP is less than $10^{-3}$. Hence, the obtained bounds can be employed as a useful tool for the evaluation of the SEP performance of ReMO for different setups at a sufficiently high SNR.

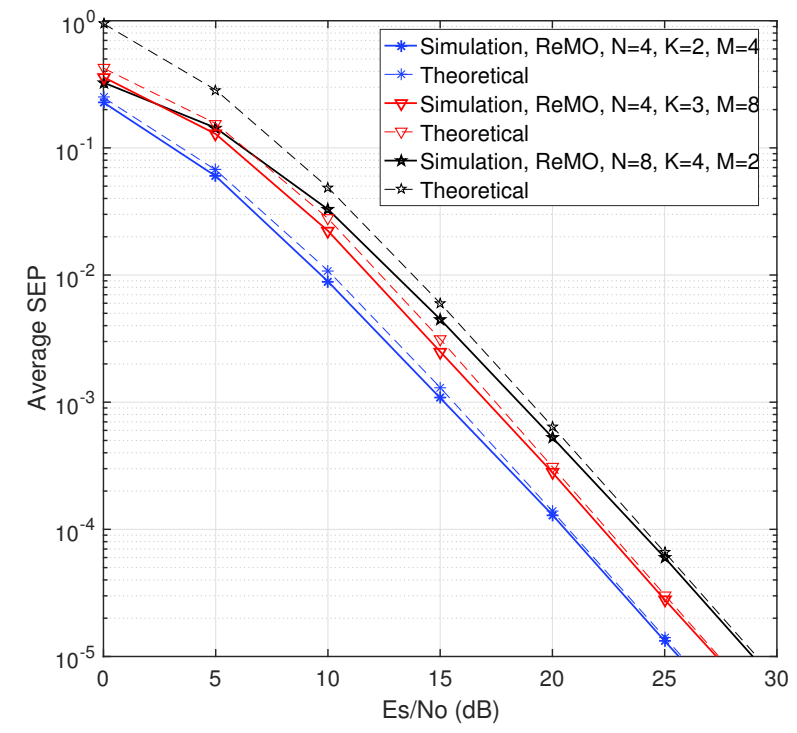

Fig. 2. Comparison between the theoretical bounds and the simulation curves on the average SEP of ReMO when $N \in\{4,8\}, K \in\{2,3,4\}$ and $M \in$ $\{2,4,8\}$.

\section{The impact of $K$ on the average SEP}

Fig. 3 investigate the impact of $K$ on the SEP of ReMO for a given spectral efficiency of $0.875 \mathrm{bps} / \mathrm{Hz}$. It is shown via Fig. 3 that the ReMO scheme of $K=2$ provides the best performance, with SNR gains of more than $1 \mathrm{~dB}$ over the scheme of $K=3$ and $3 \mathrm{~dB}$ over the remaining schemes of $K>3$. This confirms our conclusion at the end of section III that $K$ should not be too much greater than 2, meaning that the difference between the achievable diversity order in the index and frequency domains should be as small as possible. Fig. 3 also shows that the SEPs of ReMO schemes with $K \leq N / 2=$ 4 are always better than that of schemes with $K>N / 2=$ 4. This once again successfully verifies another conclusion presented at the end of section III.

\section{$D$. The impact of $M$ on the average SEP for large $K$}

We verify the effect of the PSK constellation order $M$ on the SEP of ReMO with large $K$ via Fig. 4 . It is shown in this figure that the SEPs are quite similar when $M=2,4,8$, especially in the high SNR regions $(\geq 15 \mathrm{~dB})$, the SEP of 3 these schemes are nearly the same. This observation helps us confirm the conclusion at the end of section III that for large 


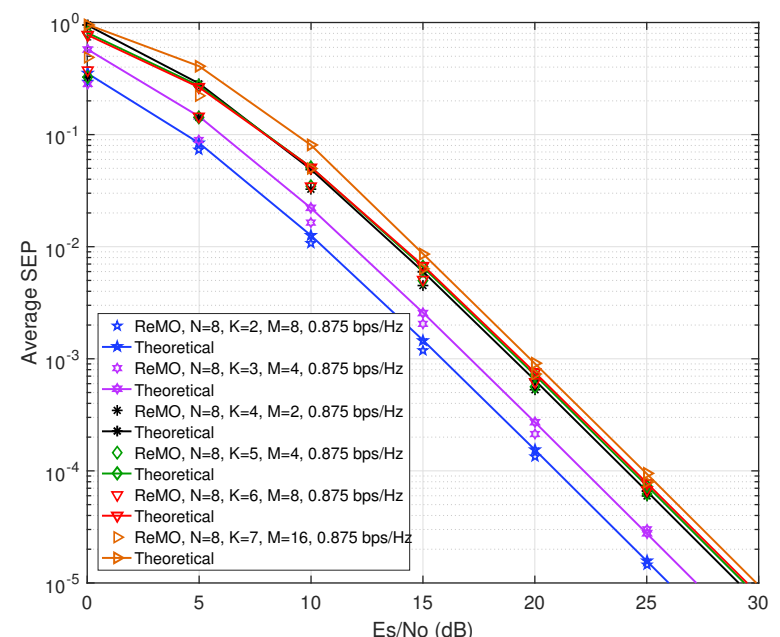

Fig. 3. Impact of $K$ on the average SEP of ReMO when $N=8, K \in$ $\{2,3,4,5,6,7\}$, and $M \in\{2,4,8,16\}$.

$K$, the SEP of ReMO mainly depends on the accuracy of the index detection i.e., the PEP. The significant difference only appears when $M$ increases to 16 or more, however, in the very high SNR regions the SEP with $M=16$ still tends to the SEP of the former schemes with smaller values of $M$.

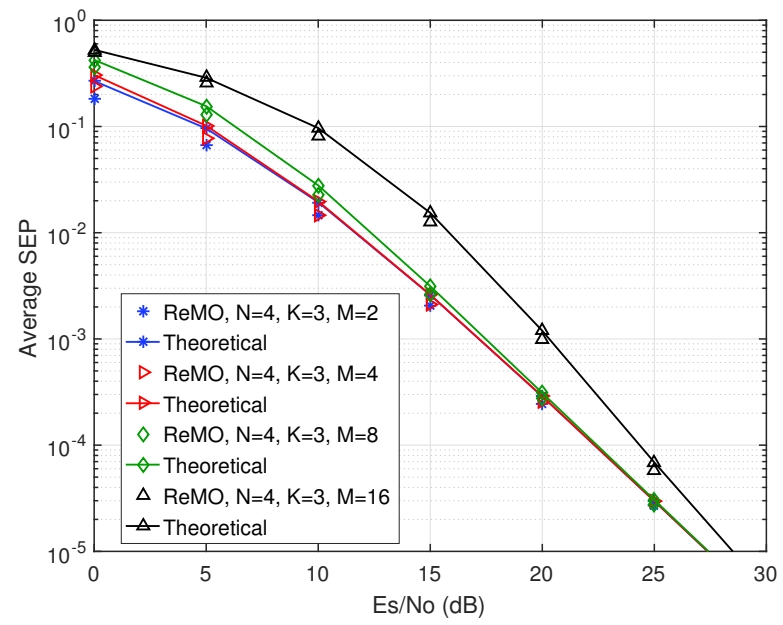

Fig. 4. Impact of $M$ on the average SEP of ReMO when $N=4, K=3$, and $M \in\{2,4,8,16\}$.

\section{Conclusions}

In this paper, we have proposed the new MCIK-OFDM scheme, called repeated MCIK-OFDM (ReMO), which has significantly improved the SEP performance over the classical OFDM and MCIK-OFDM at the same spectral efficiency. The proposed scheme has taken into account the opportunity of exploiting both the frequency and index diversity gain by repeatedly mapping the same $M$-ary symbol to all active sub-carriers and their indices. For the performance analysis, we have derived a novel expression for the symbol error probability, which provides an insight into the achievable diversity order as well as error performance. Based on this useful expression, the effect of ReMO parameters on the SEP performance can be clearly addressed in designing various concepts for future MCIK-OFDM systems.

\section{REFERENCES}

[1] E. Basar, U. Aygolu, E. Panayirci, and H. V. Poor, "Orthogonal frequency division multiplexing with index modulation," IEEE Transactions on Signal Processing, vol. 61, no. 22, pp. 5536-5549, Nov 2013.

[2] Y. Ko, "A tight upper bound on bit error rate of joint OFDM and multicarrier index keying," IEEE Communications Letters, vol. 18, no. 10, pp. 1763-1766, Oct 2014

[3] B. Zheng, F. Chen, M. Wen, F. Ji, H. Yu, and Y. Liu, "Lowcomplexity ML detector and performance analysis for OFDM with in-phase/quadrature index modulation," IEEE Communications Letters, vol. 19, no. 11, pp. 1893-1896, Nov 2015.

[4] E. Basar, "OFDM with index modulation using coordinate interleaving," IEEE Wireless Communications Letters, vol. 4, no. 4, pp. 381-384, Aug 2015.

[5] L. Wang, Z. Chen, Z. Gong, and M. Wu, "Space-frequency coded index modulation with linear-complexity maximum likelihood receiver in the MIMO-OFDM system," IEEE Signal Processing Letters, vol. 23, no. 10, pp. 1439-1443, Oct 2016.

[6] E. Basar, "Multiple-input multiple-output OFDM with index modulation," IEEE Signal Processing Letters, vol. 22, no. 12, pp. 2259-2263, Dec 2015.

[7] E. Chatziantoniou, J. Crawford, and Y. Ko, "A low complexity detector with MRC diversity reception for MCIK-OFDM," in 2016 IEEE 27th Annual International Symposium on Personal, Indoor, and Mobile Radio Communications (PIMRC), Sept 2016, pp. 1-5.

[8] R. Fan, Y. J. Yu, and Y. L. Guan, "Generalization of orthogonal frequency division multiplexing with index modulation," IEEE Transactions on Wireless Communications, vol. 14, no. 10, pp. 5350-5359, Oct 2015.

[9] T. Mao, Z. Wang, Q. Wang, S. Chen, and L. Hanzo, "Dual-mode index modulation aided OFDM," IEEE Access, vol. 5, pp. 50-60, 2017.

[10] T. Mao, Q. Wang, and Z. Wang, "Generalized dual-mode index modulation aided OFDM," IEEE Communications Letters, vol. PP, no. 99 , pp. $1-1,2016$.

[11] H. Zhang, L. L. Yang, and L. Hanzo, "Compressed sensing improves the performance of subcarrier index-modulation-assisted OFDM," IEEE Access, vol. 4, pp. 7859-7873, 2016.

[12] N. Ishikawa, S. Sugiura, and L. Hanzo, "Subcarrier-index modulation aided OFDM - will it work?” IEEE Access, vol. 4, pp. 2580-2593, 2016.

[13] C. Bockelmann, N. Pratas, H. Nikopour, K. Au, T. Svensson, C. Stefanovic, P. Popovski, and A. Dekorsy, "Massive machine-type communications in 5g: physical and MAC-layer solutions," IEEE Communications Magazine, vol. 54, no. 9, pp. 59-65, September 2016.

[14] A. Rico-Alvarino, M. Vajapeyam, H. Xu, X. Wang, Y. Blankenship, J. Bergman, T. Tirronen, and E. Yavuz, "An overview of 3GPP enhancements on machine to machine communications," IEEE Communications Magazine, vol. 54, no. 6, pp. 14-21, June 2016.

[15] M. K. Simon and M.-S. Alouini, Digital communication over fading channels, ser. Wiley series in telecommunications and signal processing. Hoboken, N.J. Wiley-Interscience, 2005.

[16] J. Proakis, Digital Communications, ser. Electrical engineering series. McGraw-Hill, 2001. 